\title{
Computer-Aided Brain Tumor Diagnosis: Performance Evaluation of Deep Learner CNN Using Augmented Brain MRI
}

\author{
Asma Naseer $(\mathbb{D}){ }^{1}$ Tahreem Yasir $\left(\mathbb{D},{ }^{1}\right.$ Arifah Azhar $(\mathbb{D}),{ }^{1}$ Tanzeela Shakeel ${ }^{D}{ }^{1}$ \\ and Kashif Zafar $\mathbb{D D}^{2}$ \\ ${ }^{1}$ University of Management and Technology, Lahore, Pakistan \\ ${ }^{2}$ National University of Computer and Emerging Sciences, Lahore, Pakistan \\ Correspondence should be addressed to Asma Naseer; asma.naseer@umt.edu.pk
}

Received 20 February 2021; Revised 6 April 2021; Accepted 31 May 2021; Published 14 June 2021

Academic Editor: Richard H. Bayford

Copyright ( 2021 Asma Naseer et al. This is an open access article distributed under the Creative Commons Attribution License, which permits unrestricted use, distribution, and reproduction in any medium, provided the original work is properly cited.

\begin{abstract}
Brain tumor is a deadly neurological disease caused by an abnormal and uncontrollable growth of cells inside the brain or skull. The mortality ratio of patients suffering from this disease is growing gradually. Analysing Magnetic Resonance Images (MRIs) manually is inadequate for efficient and accurate brain tumor diagnosis. An early diagnosis of the disease can activate a timely treatment consequently elevating the survival ratio of the patients. Modern brain imaging methodologies have augmented the detection ratio of brain tumor. In the past few years, a lot of research has been carried out for computer-aided diagnosis of human brain tumor to achieve $100 \%$ diagnosis accuracy. The focus of this research is on early diagnosis of brain tumor via Convolution Neural Network (CNN) to enhance state-of-the-art diagnosis accuracy. The proposed CNN is trained on a benchmark dataset, $\mathrm{BR} 35 \mathrm{H}$, containing brain tumor MRIs. The performance and sustainability of the model is evaluated on six different datasets, i.e., BMI-I, BTI, BMI-II, BTS, BMI-III, and BD-BT. To improve the performance of the model and to make it sustainable for totally unseen data, different geometric data augmentation techniques, along with statistical standardization, are employed. The proposed CNN-based CAD system for brain tumor diagnosis performs better than other systems by achieving an average accuracy of around $98.8 \%$ and a specificity of around 0.99 . It also reveals $100 \%$ correct diagnosis for two brain MRI datasets, i.e., BTS and BD-BT. The performance of the proposed system is also compared with the other existing systems, and the analysis reveals that the proposed system outperforms all of them.
\end{abstract}

\section{Introduction}

The brain is considered one of the most important organs, being responsible for memory, emotions, vision, motor skills, reactions, respiration, and a lot of other regulating functions of the human body. These functions are severely affected if some tumor starts growing inside the brain. This tumor is either the primary brain tumor which starts growing inside the brain, which is the growth of brain tissues itself, or a metastasis brain tumor which starts in some other part of the body and spreads to the brain [1]. Diagnosis of a tumor in the brain is very challenging as compared to a tumor from any other part of the human body. As the brain is filled with the blood-brain barrier (BBB), so the ordinary radioactive indicators are not able to capture the hyperactivity of tumor cells [1]. Therefore, Magnetic Resonance Imagery (MRI) and Computed Tomography (CT) scans are regarded as the best diagnostic tracers to capture disruption in BBB.

For different age groups, almost 7 to 11 persons per 100,000 get brain tumor annually $[2,3]$. The Global Burden of Disease (GBD) reports almost 227,000 deaths per year due to this deadly disease. Besides, almost 7.7 million persons who survived are spending a life with disability adjustment [3]. Early detection of brain tumor not only helps in saving lives but also helps in eliminating the chance of disabilities. With early diagnosis, there will be lesser manipulation and surgical removal from the brain which is the most sensitive part of the body [4]. The manual diagnosis of the disease requires a radiologist to record a $3 \mathrm{D}$ image for initial insight. Then, an expert doctor is engaged for image examination and treatment 
planning. Unfortunately, the studies [5] investigating the accuracy of manual brain tumor diagnosis reports a disagreement between expert reviewers. The maximum agreement between the experts for manual diagnosis of brain tumor is reported between $90 \%$ and $95 \%$. For mixed categories of tumor, mixed glioma, and medulloblastoma, the disagreement between the experts further decreases to $77 \%$ and $58 \%$, respectively [5].

With the evolution of medical imaging technologies (MRI, CT scan, etc.) and the development in digital image processing, computer-aided diagnosis (CAD) of tissues and tumors has increased [6]. For such diagnosis systems, MRI is preferred as there is no risk of ionising radiation, and it can detect blood flow in veins accurately [7]. In the past few years, different techniques have been proposed for CAD systems for brain tumor, such as fused vectors [8], Support Vector Machine (SVM) [9, 10], transfer learning [11], and deep networks (NWs) [12]. With the recent developments in deep NWs, Convolution Neural Network (CNN) has been widely used for different CAD systems [13-16].

$\mathrm{CNN}$ is a sequence of multiple layers where each layer extracts features and transforms a complex input into an activation form, using partial differential functions. The layers are built on the top of each other. CNN architecture has three basic layers, i.e., a convolution layer, a pooling layer, and a fully connected layer. Where the convolution layer extracts features gradually, pooling layers downsample along the spatial domain, and the fully connected layer classifies. A vanishing gradient problem may rise when small numbers appear while computing gradients. To avoid vanishing gradient difficulty, a Rectified Linear Unit (ReLU) layer is also added after each convolution layer, as an element-wise activation function. Some other CNN layers are the input layer, the dropout layer, the output layer, and the network in network layer $[17,18]$.

In this article, a computer-aided brain tumor diagnosis tool is proposed to examine brain MRIs and provide an early diagnosis with improved performance. For this proposed CAD system, CNN is trained on the BR35H::Brain Tumor Detection 2020 dataset [19], and its performance is evaluated for six different brain tumor MRI datasets [20-25]. The trained CNN model achieves $100 \%$ accuracy for two datasets, i.e., Brain Tumor Segmentation (BTS) [24] and BDBrainTumor (BD-BT) [23]. For the performance evaluation of the model, different statistical evaluation methods are used including sensitivity, specificity, precision, recall, $f_{\text {measure' }}$ false positive (FP) ratio and Receiver Operating Characteristic (ROC) curve.

The major contributions of this research are as follows:

(i) An outperforming CNN-based computer-aided brain tumor diagnosis system for an early and reliable detection of brain tumor to assist in the rapid treatment planning

(ii) A technique consisting of preprocessing on images, feature extraction, reduction of feature space, and finally classification of images into positive and negative diagnoses of brain tumor (iii) Improvement in $\mathrm{CNN}$ performance by applying geometrical and statistical data augmentation techniques on brain tumor MRI

(iv) Evaluation of the power of $\mathrm{CNN}$ by classifying totally unseen data, consisting of the six latest brain tumor MRI datasets

(v) Producing up to $100 \%$ accuracy by the trained CNN model

(vi) Achieving a high average sensitivity, i.e., 0.99 for all the datasets

(vii) A comprehensive comparative analysis with the other existing systems

The paper is arranged in such a way that Section 2 presents related work, Section 3 describes proposed methodology, Section 4 portrays results and discussion, and Section 5 concludes the research along with the future work.

\section{Related Work}

CNN has been widely used for solving different problems in different areas $[17,18]$ but, for the processing of images for health applications, its performance is remarkable. A lot of research exists in which CAD-based diagnosis of diseases is proposed. For the detection of brain tumor, a CNN with a neutrosophic is explored [26]. In this hybrid technique, features are extracted by CNN, and for classification, Support Vector Machine (SVM) and K-Nearest Neighbors (KNN) are used. The system is trained and tested on only 160, half negative and half positive, images. Using fivefold cross-validation, $95.62 \%$ accuracy is achieved by the proposed technique.

In another research, brain tumor is detected by using both handcrafted features and the features extracted by the deep learner [8]. In the proposed system, a transfer learning model acquires features while shape and texture are extracted manually. For classification, entropy and fused vectors are fed to the classifier. In another research, brain tumor is classified by using CNN and transfer learning [13]. For this experiment, pretrained GoogLeNet is used to extract features. For classification, already proven classifiers are used. By using fivefold cross-validation, $98 \%$ accuracy is achieved. $\mathrm{CNN}$ is trained on augmentation of extensive data for brain tumor classification [15]. In the proposed system, tumor area is segmented by using a deep learning technique. The research uses a pretrained CNN model and evaluates the performance of the system on original as well as augmented data.

In the proposed system, brain tumor MRIs are used to train CNN [16]. In this research, $\mathrm{CNN}$ architecture is designed by using a hypercolumn technique. An attention module identifies the area of interest before transferring it to CNN layers. The proposed system achieves $96.05 \%$ accuracy. $\mathrm{CNN}$ is also used for the segmentation of brain tumor in MRI [14]. The results of a clustering algorithm, traditional classifiers, and CNN are compared. Traditional classifiers include Multilayer Perceptron (MLP), Support Vector Machine (SVM), Logistic Regression, K-Nearest Neighbor (KNN), Random Forest, and Naïve Bayes. The performance 
of CNN, with $97.87 \%$ accuracy, is reported as the best among all the classifiers. For combining texture and structural information in the four MRI sequences, a fusion process is implemented for brain tumor detection [27]. A fusion process uses Discrete Wavelet Transformation (DWT). By using the Daubechies wavelet, more information from the tumor region is extracted. After preprocessing, $\mathrm{CNN}$ is used for classification of tumor and nontumor regions. As per results, fused images reveal better performance. In another research, six CNN models are trained for brain tumor detection [9]. The architecture of the CNN models is defined on the basis of different hyperparameters. The results show a better performance by deep learning models as compared to the conventional methods. In another similar approach, different architectures for $\mathrm{CNN}$ models are designed for classifying benign tumor [10]. The accuracy for different models is reportedly between $96 \%$ and $99 \%$.

In a study, normal brain tissues are differentiated from brain tumor and pseudobrain tumor by using LSTM [28]. Different augmentation techniques are applied on an MRI signal dataset for training stacked Bi-LSTM. Using 5-fold cross-validation, average accuracy achieved by the proposed technique is $91.46 \%$. A multiscale Deep CNN [29] is proposed which can analyse tumor MRIs and classify them into glioma, meningioma, and pituitary tumor. The performance of the proposed model is evaluated on an MRI image dataset consisting of 3,064 images. Classification accuracy of the proposed CNN is reported as $97.3 \%$. Deep network ResNet-50 is trained on 3,064 brain MR images taken from three brain MRI datasets [30]. The performance of the model is evaluated with the help of a key performance matrix. The proposed model achieves $97.08 \%$ average accuracy for nonaugmented data and $97.48 \%$ average accuracy for augmented data. In another study, eight CNN models [31] are developed and trained on brain MRI for a CAD system of brain tumor. CNN models reveal accuracy between $90 \%$ and $99 \%$. A 3D CNN model is proposed to extract features from brain MRIs [32]. The features extracted by $\mathrm{CNN}$ are provided to a correlation-based model for optimum feature selection, and a feed-forward ANN is used for classification. The accuracy achieved by the proposed technique is $92.67 \%, 96.97 \%$, and $98.32 \%$, for three different datasets.

\section{Proposed Methodology}

The focus of the current research is on computer-aided diagnosis of brain tumor by feeding brain tumor MRIs to CNN. Using labelled data, CNN extracts features and learns to classify images as positive or negative diagnosis of brain tumor. This supervised model of CNN uses preprocessed images for a better performance. The main phases of research include, gathering the latest brain tumor image dataset, preprocessing on images, gradual and incremental training of the model, and finally performance evaluation by testing the model on six different unseen MRI datasets.

3.1. Datasets. In the brain MRI images, brain tissues can be represented by either $\mathrm{T} 1$ or $\mathrm{T} 2$ relaxation time. $\mathrm{T} 1-$ weighted images use short Repetition Time (TR) and Time
TABLE 1: Time in milliseconds, taken by T1-weighted image scanning and T2-weighted image scanning.

\begin{tabular}{lcc}
\hline & $\begin{array}{c}\text { T1-weighted } \\
\text { (short TE and TR) }\end{array}$ & $\begin{array}{c}\text { T2-weighted } \\
\text { (long TE and TR) }\end{array}$ \\
\hline TE & $14 \mathrm{~ms}$ & $90 \mathrm{~ms}$ \\
TR & $500 \mathrm{~ms}$ & $4000 \mathrm{~ms}$ \\
\hline
\end{tabular}

to Echo (TE) while T2-weighted images use longer TR and TE times. The time taken in milliseconds by T1- and T2weighted scanning is given in Table 1 . The training data that is used in this research contains both T1-weighted and T2weighted images, while the datasets used for testing contain either T1-weighted images or T2-weighted images. The sample T1-weighted and T2-weighted images are described in Figures 1(a) and 1(b), respectively.

The signals generated by loop coils of the MRI device are digitized by Fast Fourier Transformation (FFT) which provides real value, imaginary value, magnitude, and phase. All the datasets, used in this research, are based on the magnitude of FFT.

The performance of machine learning and deep learning techniques highly depends on the dataset and its size. The uniqueness of this work is to train the model on a small dataset in such a way that it can be sustainable for unseen data which is the exact target of these learning systems. Therefore, in this research, almost only $28 \%$ data is used for training the model while the rest of the data is used for testing to assure the robustness of the proposed CAD system.

3.1.1. Training Dataset. For training CNN, the BR35H::Brain Tumor Detection 2020 (BR35H) [19] dataset is used which contains 255 negative and 255 positive MRIs of brain tumor. $90 \%$ of the images from this dataset are used for training the model. The dataset contains both T1-weighted and T2weighted image sequences. The usability rating of this dataset is reported as 7.5 [19]. Data usability rating is calculated on the basis of licensing, tagging, overview of data, and its description, ease, maintainability assurance, machine readable file formats, metadata, and availability of public kernel.

3.1.2. Testing Datasets. For the performance evaluation of $\mathrm{CNN}$, six totally unseen datasets are used including Brain MRI Images for Brain Tumor Detection (BMI-I) [21], Brain Tumor Image Dataset (BTI) [25], Brain MRI Images for Brain Tumor Detection (BMI-II) [22], Brain Tumor Segmentation (BTS) [24], Brain MRI Images for Brain Tumor Detection (BMI-III) [20], and BD-BrainTumor (BD-BT) [23]. An overview of these datasets is given in Table 2.

(1) BMI-I. The BMI-I dataset contains, in total, 171 images out of which 86 images are positive for brain tumor and 85 images are negative. The images in this dataset are T1weighted, and the usability of this dataset is reported as 5.0.

(2) BTI. The BTI dataset consists of 20 images with $50 \%$ positive and 50\% negative class labels. The images in this dataset are $\mathrm{T} 2$-weighted, and the usability of this dataset is reported as 4.4 . 


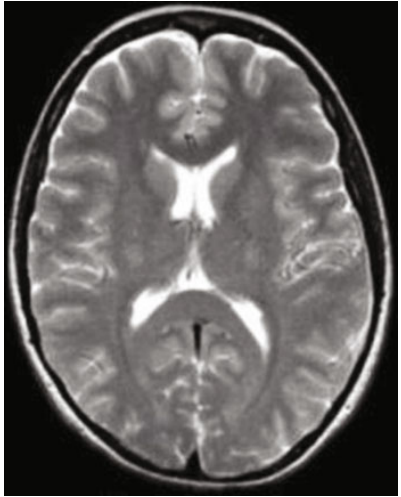

(a) T1-weighted MRI with darker CRF

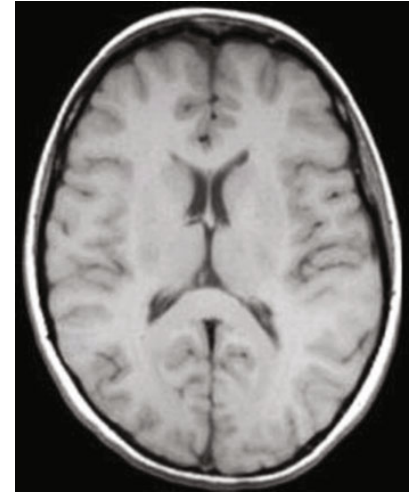

(b) T2-weighted MRI with brighter CRF

FIGURE 1: Samples of T1-weighted and T2-weighted brain MRI with different intensity values of cerebrospinal fluid (CRF).

TABLE 2: Brain MRI datasets.

\begin{tabular}{|c|c|c|c|c|c|}
\hline & & Brain tumor images datasets & Positive & Negative & Total \\
\hline Training & $\mathrm{BR} 35 \mathrm{H}$ & BR35H::Brain Tumor Detection 2020 & 255 & 255 & 510 \\
\hline \multirow{7}{*}{ Testing } & BMI-I & Brain MRI Images for Brain Tumor Detection & 86 & 85 & 171 \\
\hline & BTI & Brain Tumor Image Dataset & 10 & 10 & 20 \\
\hline & BMI-II & Brain MRI Images for Brain Tumor Detection & 86 & 6 & 92 \\
\hline & BTS & Brain Tumor Segmentation & 70 & 70 & 140 \\
\hline & BMI-III & Brain MRI Images for Brain Tumor Detection & 86 & 85 & 171 \\
\hline & \multirow{2}{*}{ BD-BT } & BD-BrainTumor & 671 & 0 & 671 \\
\hline & & Total & 1264 & 511 & 1775 \\
\hline
\end{tabular}

(3) BMI-II. There are 92 images which are taken from the BMI-II dataset. 86 images are labelled as positive and 6 images are labelled as negative. The images in this dataset are T1-weighted, while the usability ranking of this dataset is 3.8 .

(4) BTS. 140 images from the BTS dataset, used in this research, consists of the same number of positive and negative images. The images in this dataset are T2-weighted, and the usability of this dataset is reported as 3.1.

(5) BMI-III. BMI-III contains 86 positive and 85 negative images. The images are T1-weighted, and the usability of this dataset is 1.3 .

(6) BD-BT. 671 images, all positive, are used from BD-BT dataset. The images in this dataset are T2-weighted, while the usability ranking of this dataset is reported as 2.5 .

3.2. Data Augmentation and Preprocessing. In this research, a total of 1,775 images were used for training and testing. All the images are preprocessed before feeding them to $\mathrm{CNN}$, as described in Figure 2. At first, these images are converted into single-channel images, known as greyscale images. After colour data augmentation, geometric data augmentation including scaling, flipping, and rotation is applied. As the original images are in different sizes, so they are rescaled to a size of $256 \times 256$. To make CNN perform dynamically for different datasets, image reflections, in both horizontal and vertical dimensions, are generated by using equation (1) and equation (2). The effects of such reflections can be seen in Figure 3.

$$
\begin{aligned}
& \mathrm{BT}_{\mathrm{h}}(x, y)=\mathrm{BT}(-x, y), \\
& \mathrm{BT}_{\mathrm{v}}(x, y)=\mathrm{BT}(x,-y),
\end{aligned}
$$

where BT is the original MRI of the brain, $\mathrm{BT}_{\mathrm{h}}$ is the horizontal reflection, and $\mathrm{BT}_{\mathrm{v}}$ is the vertical reflection.

After getting the reflected images, two more types of images are generated by rotating the original images at $45^{\circ}$ and $90^{\circ}$. For rotations, equation (3) and equation (4) are used. The effects of these rotations are illustrated in Figure 4.

$$
\begin{aligned}
R_{\theta} & =\left[\begin{array}{cc}
\cos \theta & -\sin \theta \\
\sin \theta & \cos \theta
\end{array}\right], \\
\mathrm{BT}_{\mathrm{r}} & =\mathrm{BTR}_{\theta},
\end{aligned}
$$

where $\mathrm{BT}$ is the original MRI of the brain, $\mathrm{BT}_{\mathrm{r}}$ is the rotated image, $R_{\theta}$ is the rotation matrix, and $\theta$ is set as $45^{\circ}$ and $90^{\circ}$.

As the brain tumor MRI datasets used in this research are from seven different sources and are in different formats, so further preprocessing is applied before feeding these images 


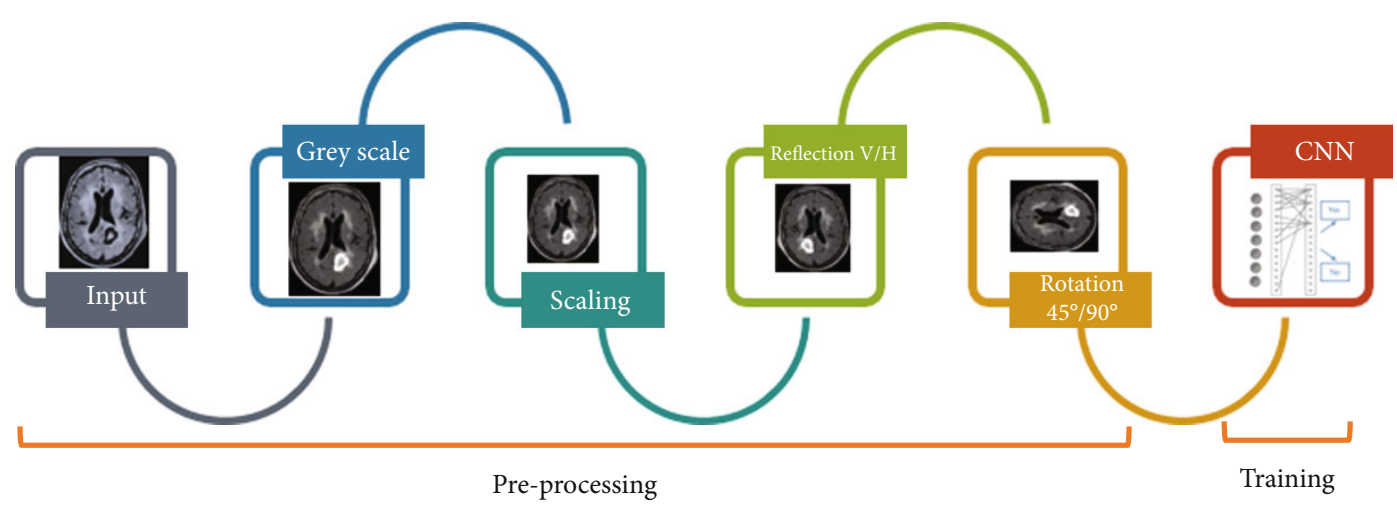

Figure 2: Preprocessing steps: greyscale conversion, scaling, rotation, and reflection.
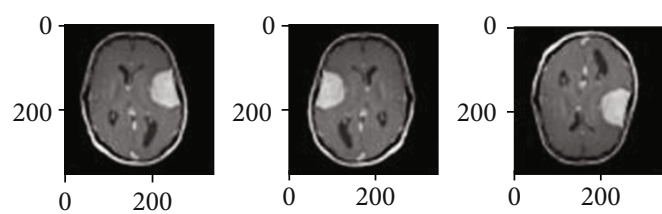

FIgURE 3: Original image, image with horizontal reflection, and image with vertical reflection.
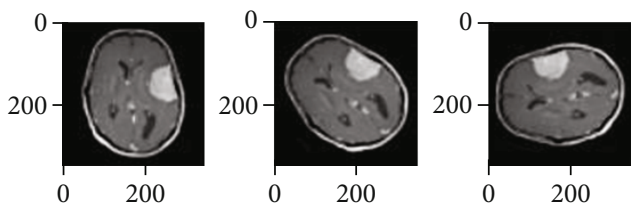

FIGURE 4: Original image, image with $45^{\circ}$ degree rotation, and image with $90^{\circ}$ degree rotation.

to CNN. To harmonize these images, standardization is performed by using equation (5), equation (6), and equation (7). With the help of the standardization process, all the images get 0 as mean $\mu$ and 1 as standard deviation $\sigma$. Turning $\mu$ into zero makes different datasets comparable. On the other hand, $\sigma$ with a value of 1 , makes the data distribution comparable to a normal distribution.

$$
\begin{aligned}
\mu & =\frac{\sum_{x=1}^{M} \sum_{y=1}^{N} \mathrm{BT}_{x, y}}{M \times N}, \\
\sigma & =\sqrt{\frac{1}{M \times N} \sum_{x=1}^{M} \sum_{y=1}^{N}\left(\mathrm{BT}_{x, y}-\mu\right)^{2},} \\
\mathrm{BT}_{\mathrm{s}} & =\mathrm{BT}-\frac{\mu}{\sigma},
\end{aligned}
$$

where BT is the brain tumor image, $M$ and $N$ are its dimensions, $\mathrm{BT}_{\mathrm{s}}$ is the standardized brain tumor image, $\mu$ is mean, and $\sigma$ is standard deviation.

Finally, all the images with negative labels are mapped to the value of 0 , and the ones with a positive label are mapped to the value of 1 , for the supervised training of $\mathrm{CNN}$.
3.3. Convolution Neural Network (CNN) Architecture. The architecture of $\mathrm{CNN}$ is defined sequentially, and the model is built layer by layer. The first layer is the input layer, consisting of a size defined by the input images. The next layer is the $2 \mathrm{D}$ convolution layer with 32 filters and a $2 \times 2$ kernel. A Rectified Linear Unit (ReLU) computes cheaply and converges quickly. To avoid the "dyingReLU" problem, i.e., zero ReLU for negative values, LeakyReLU, with alpha set as 0.001 , is added on the top of the convolution layer. After this layer, the dropout layer is added with a dropout rate set as 0.3 . Once this architecture is defined, a $2 \mathrm{D}$ convolution layer is again created using a kernel function with a size of $3 \times 3$ followed by LeakyReLU and dropout layers with the previously defined parameters. After these layers, another 2D convolution layer is added with the number of filters set as per the image size. After that, a pooling layer is added to reduce and summarise the feature map by downsampling. To prevent the CNN model from overfitting, a dropout layer is added to the network after the pooling layer. After this layer, a flattening technique is defined for the output of the network followed by two fully connected dense layers with units 12 and 1. LeakyReLu and sigmoid are used as activation functions in these fully connected layers, respectively. The architecture of CNN implemented in this research is illustrated in Figure 5.

Convolution operations are performed by applying equation (8), and for nonlinearity, ReLU layers are implemented with equation (9).

$$
\begin{aligned}
& X[i, j]=(w \times x)[i, j]=\sum_{s=-M}^{M} \sum_{t=-N}^{N} x[i+s, j+t] w[s, t], \\
& f(x)= \begin{cases}x, & \text { if } x \geq 0, \\
\alpha x, & \text { otherwise, }\end{cases}
\end{aligned}
$$

where $x$ is the input image of brain tumor, $w$ is the kernel or convolution operator, $X$ is the feature map of processed data, kernel size is $M \times N$, and $i$ and $j$ are the row and the column at $i$ th and $j$ th position of input $x$. The value of $\alpha$ is set as 0.001 .

The dropout layer regularises the deep learners to avoid overfitting. This layer can be applied on a fully connected layer or a convolutional layer. The effect on a fully connected 


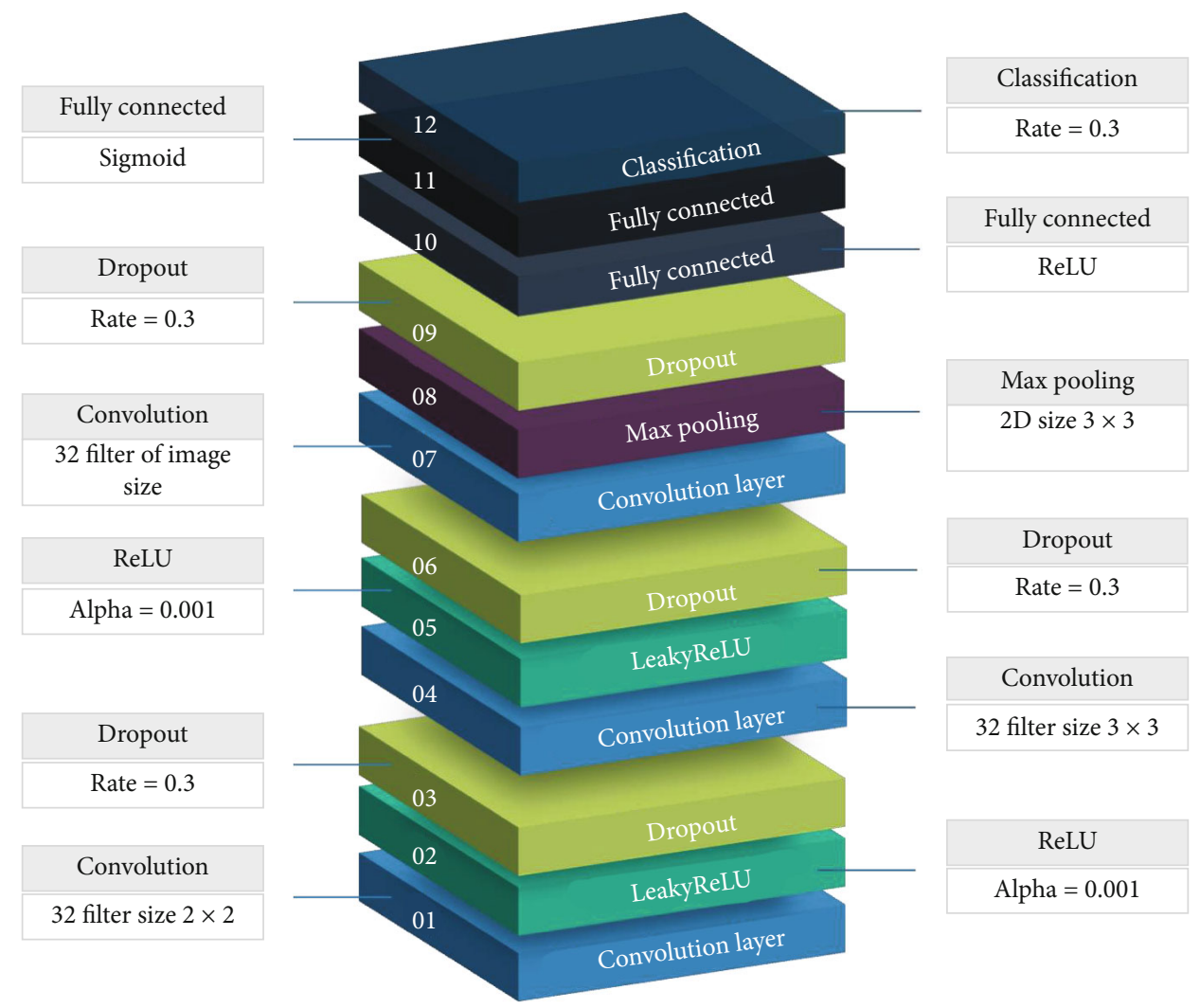

FIGURE 5: Layers of the proposed convolution neural network architecture.

layer would be in the shape of dropping out neurons to avoid overfitting, while the effect on the convolutional layer would be in the shape of adding noise into the feature maps. In the preprocessing phase, normalisation and data augmentation are already applied on the training data, hence, the dropout layer is not applied on fully connected layers, rather it is applied on the convolution layer to generate effects on feature maps. In this way, the effect of the augmented data on overfitting is evaluated without dropping out neurons.

3.3.1. Padding and Stride. Due to convolution operations at convolutional layers, some pixels from the boundary of the images are lost, thus resulting in different input and output image sizes. The loss of pixels depends on kernel size. If the size of kernel is $(n \times m)$ and $n$ is even, then $(n / 2)$ rows from the top and bottom of the image will be lost. In case $n$ is odd, then the loss will be of $(n / 2)-1$ rows from the top and bottom. The same is true for the first and last $m$ number of columns. To overcome this loss, an elementary solution is to add extra pixels all over the boundary. In the proposed $\mathrm{CNN}$, zero padding is used, considering the size of the kernel. In the case of even values of $m$ and $n,(n / 2) \times(m / 2)$ zero padding is applied, and for odd values of $m$ and $n,((n / 2)-1) \times$ $((m / 2)-1)$ zero padding is applied around the image boundary. For convolving with the image, the kernel window slides on the whole image step by step. Stride is the component of $\mathrm{CNN}$ which decides the size of step. In the proposed CNN, the value of stride is set as 1 , for both rows and columns.
3.4. Training. After preprocessing the images and designing all the layers of the CNN architecture, the deep network $\mathrm{CNN}$ is trained on rescaled and preprocessed original images along with their reflected and rotated set of images from dataset BR35H. For training the model, sigmoid activation function, given in equation (10), is used as an optimiser due to its nature of smoother output while observing smaller changes in the input. The learning rate of the model is set as 0.001 .

$$
f(x)=\sigma(x)=\frac{1}{1+e^{-x}}
$$

For training CNN for binary classification of brain tumor MRIs, cross entropy, given in equation (11), is used as a loss function.

$$
\mathrm{CE}=-\sum_{i=1}^{c^{\prime}=m} l_{i} \log \left(s_{i}\right)=l_{1} \log \left(s_{1}\right)-\left(1-l_{1}\right) \log \left(1-s_{1}\right),
$$

where $l_{i}$ and $s_{i}$ are the CNN scores for each positive and negative class, while the value of $m$ is 2 (binary classifier).

Instead of training CNN in one go, a step by step gradual training is performed in six phases, and its performance on the validation set is evaluated before entering in the testing phase. For validation, $10 \%$ of data from the BR35H dataset 
TABLE 3: Loss and accuracy trends during the gradual training of CNN model.

\begin{tabular}{lccc}
\hline No. of epochs/phase & Total epochs & Loss & Accuracy \\
\hline 10 & 10 & 0.4711 & 79.36 \\
5 & 15 & 0.3675 & 83.44 \\
10 & 25 & 0.13 & 94.34 \\
20 & 45 & 0.0098 & 98.91 \\
20 & 49 & 0.0091 & 100 \\
& 65 & 0.0077 & 100 \\
\hline
\end{tabular}

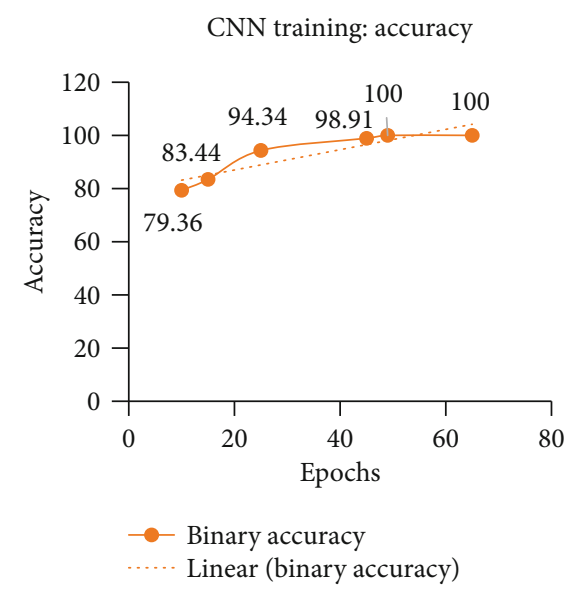

Figure 6: Training accuracy curve of the CNN model showing the gradual progress of the deep neural network.

is used after shuffling the images. At first, CNN is trained only for 10 epochs. After 10 epochs, 79.36\% binary classification accuracy is achieved on the validation set. Due to such low training accuracy, $\mathrm{CNN}$ is again trained for 5 more epochs. To enhance the training accuracy of the deep network, it is gradually trained for three more times with 10 , 20, and again 20 epochs. The increase in training accuracy with more training of the model is described in detail in Table 3. After the 49th epoch, CNN shows 100\% training accuracy on the validation set.

At each time, the training of the CNN model is made with a fixed number of epochs. The two curves of training accuracy and training loss are monitored. If the curves show an overall monotonic increase in accuracy and monotonic decrease in loss, then the model is further trained for another fixed number of epochs. The training of the CNN model is stopped when $100 \%$ training accuracy is achieved. The gradual increase in accuracy and decrease in loss during the 65 epochs of training are illustrated in Figures 6 and 7, respectively.

\section{Results and Discussion}

For this research, only one MRI dataset of brain tumor is used for training $\mathrm{CNN}$, while six datasets are used for testing the performance of the model. In this way, almost only $28 \%$ of the data is used for training and validation, while $72 \%$ of the data is used for testing. The performance of CNN is evaluated by precision, recall/sensitivity, $f_{\text {measure, }}$ and

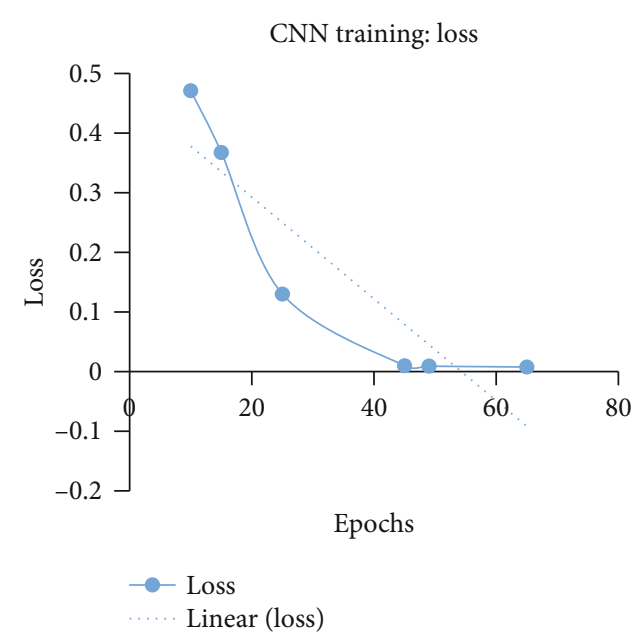

FIGURE 7: Loss curve during the training of the CNN model showing a good learning rate.

TABle 4: Precision, recall, and $F_{\text {measure }}$ with $\alpha=0.5$, for six testing datasets.

\begin{tabular}{lcccccccc}
\hline Datasets & Total & TP & TN & FP & FN & Precision & Recall & $\begin{array}{c}F_{\text {measure }} \\
\alpha=0.5\end{array}$ \\
\hline BMI-I & 171 & 86 & 80 & 0 & 5 & 1 & 0.946 & 0.973 \\
BTI & 20 & 9 & $\mathbf{8}$ & 1 & 2 & 0.9 & 0.819 & 0.86 \\
BMI-II & 92 & 86 & $\mathbf{5}$ & 0 & 1 & 1 & 0.989 & 0.995 \\
BTS & 140 & 70 & $\mathbf{7 0}$ & 0 & 0 & 1 & 1 & 1 \\
BMI-III & 171 & 86 & $\mathbf{7 9}$ & 0 & 6 & 1 & 0.935 & 0.968 \\
BD-BT & 671 & 671 & $\mathbf{0}$ & 0 & 0 & 1 & 1 & 1 \\
Total & $\mathbf{1 2 6 5}$ & $\mathbf{1 0 0 8}$ & & $\mathbf{1}$ & $\mathbf{1 4}$ & - & - & - \\
\hline
\end{tabular}

specificity as given in equation (12), equation (13), equation (14), and equation (15).

$$
\begin{aligned}
\text { Precision } & =\frac{\mathrm{TP}}{(\mathrm{TP}+\mathrm{FP})}, \\
\text { Recall } & =\text { sensitivity }=\frac{\mathrm{TP}}{(\mathrm{TP}+\mathrm{FN})}, \\
f_{\text {measure }} & =\frac{2 \times \text { precision } \times \text { recall }}{\text { precision }+ \text { recall }}, \\
\text { Specificity } & =\frac{\mathrm{TN}}{(\mathrm{TN}+\mathrm{FP})} .
\end{aligned}
$$

The accuracy of CNN for all the datasets is above 96\%, except for the dataset BTI, as described in Table 4. For the BTS and BD-BT datasets, the model has classified the brain tumor images with $100 \%$ accuracy. The performance of the model is consistent for the six datasets, excluding BTI, which contains only 20 images out of the 1265 tested images. The accuracy of each test dataset is illustrated in Figure 8.

The research also reveals that the performance of CNN is very remarkable for positive class images, as out of 1009 


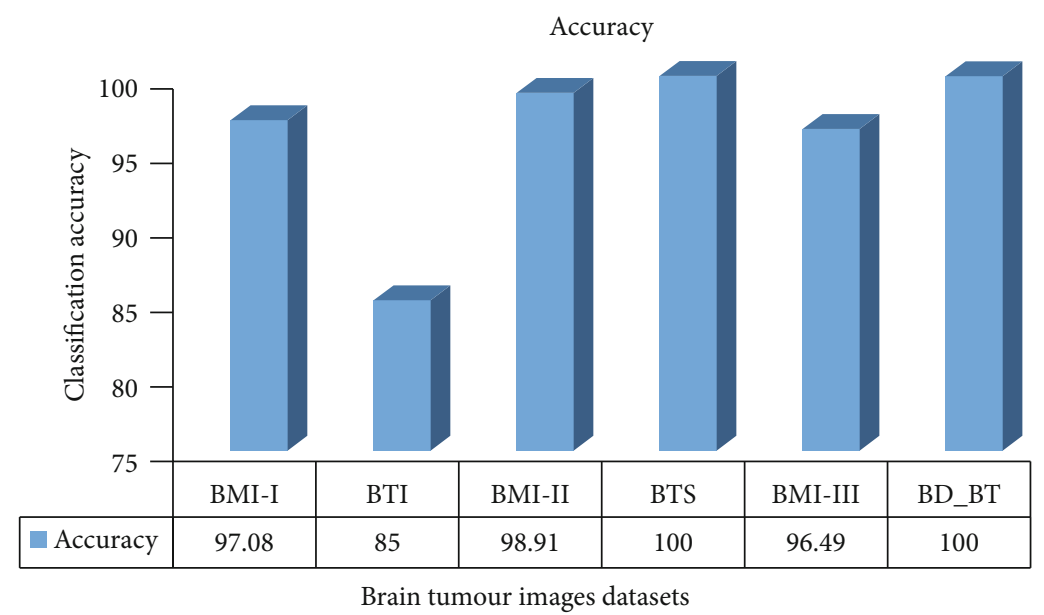

Figure 8: Accuracy of the proposed Convolution Neural Network for all the six test datasets.

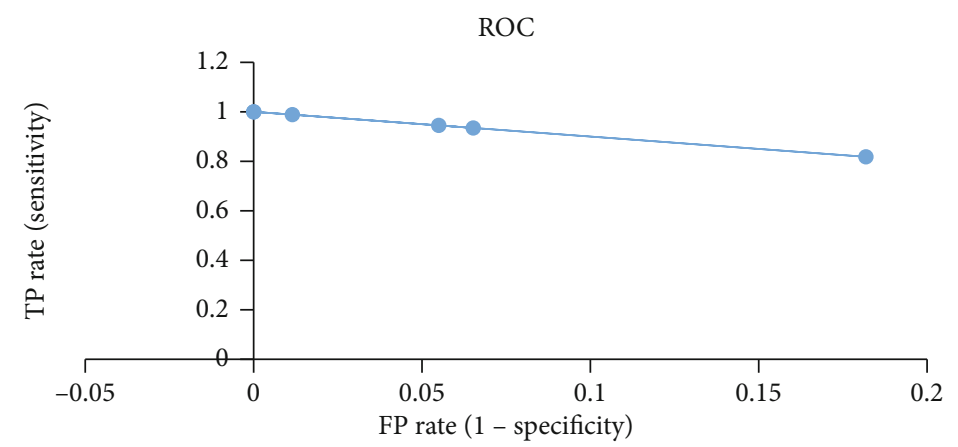

Figure 9: Receiver Operating Characteristic (ROC) curve between True Positive Rate (TPR) and False Positive Rate (FPR).

positive class images only 14 images are missclassified as negative. CNN performance for positive images is also visible in the test results of the BD-BT dataset which contains 671, all positive images, classified correctly. Even for negative class images, the performance of $\mathrm{CNN}$ is quite reliable as only 1 image, out of 242 negative images, is classified as false positive. The average accuracy of $\mathrm{CNN}$, for all the six datasets, is $98.8 \%$. For analysing TP rate vs. FP rate, ROC is also plotted in Figure 9.

The architecture of $\mathrm{CNN}$, defined in this research to diagnose brain tumor with the help of preprocessed brain MRI, is able to achieve reliable accuracy. As compared to the other latest research work $[10,13,14,16,26]$, where CNN models are trained on $80 \%$ of data and accuracy is still less than $100 \%$, the CNN model, designed and trained in this research, reveals a better performance. The model is able to achieve $100 \%$ accuracy on two datasets $[23,24]$. Even the accuracy reported by using pretrained CNN models $[13,15]$ is not better than the CNN model that is trained in this research.

The outstanding performance of $\mathrm{CNN}$ is due to different factors. The architecture of $\mathrm{CNN}$, designed in this research, contains three convolution layers. Convolution layers, the basic building blocks of the network, merge different sets by convolving images with the convolution filter, thus creating a feature map. In the proposed architecture, three layers are designed for extracting feature maps for producing more information for classification. The decision regarding hyperparameters, such as filter size and filter count, also plays a vital role in the learning phase of the network. For better learning, $\mathrm{CNN}$ is trained in a such a way that overfitting can be avoided for which an augmented data technique is used. For augmented data, different transformations such as rotation and reflection are applied to the input images. Due to data augmentation via transformations, regularisation of data is achieved which ultimately leads CNN to learn in a generic way instead of remembering only training data. Due to this factor, CNN avoids overfitting and performs better even for unseen datasets from different resources. Another factor which helped to achieve better accuracy is data cleaning, for which mean normalisation is applied to the input images. It enables $\mathrm{CNN}$ to compare different datasets and perform remarkably. Besides, dropout layers, which regularise $\mathrm{CNN}$ to avoid overfitting, are also applied on convolution layers which add noise to feature maps. Adding such an effect into feature maps makes $\mathrm{CNN}$ more robust and sustainable.

4.1. Comparative Analysis with the Other Systems. The performance of the proposed system is compared with the other most recent computer-aided brain tumor diagnosis systems. In these systems, CNN [33-37], Random Forest [38], Artificial Neural Network (ANN) [39], Deep CNN (D-CNN) [40], Support Vector Machine (SVM) [41], and Faster Region- 
TABLE 5: Comparative analysis of the proposed system with the other CAD systems.

\begin{tabular}{|c|c|c|c|c|}
\hline Reference & Technique & Training images & Testing images & Accuracy \\
\hline$[38]$ & Random Forest Classifier & 372 & 93 & $86 \%$ \\
\hline$[36]$ & $\mathrm{CNN}$ & 2451 & 613 & $91.30 \%$ \\
\hline$[35]$ & $\mathrm{R}-\mathrm{CNN}$ & 2451 & 613 & $91.66 \%$ \\
\hline$[39]$ & ANN & 160 & 40 & $92.14 \%$ \\
\hline$[34]$ & $\mathrm{CNN}$ & 222 & 56 & $93.9 \%$ \\
\hline$[33]$ & $\mathrm{CNN}$ & 400 & 100 & $96.08 \%$ \\
\hline$[37]$ & $\mathrm{CNN}$ & 2451 & 613 & $96.13 \%$ \\
\hline$[41]$ & Support Vector Machine (SVM) & 372 & 93 & $97.1 \%$ \\
\hline$[40]$ & Deep CNN (D-CNN) & 372 & 93 & $98.07 \%$ \\
\hline Proposed model & $\mathrm{CNN}$ & 510 & 1265 & $98.8 \%$ \\
\hline
\end{tabular}

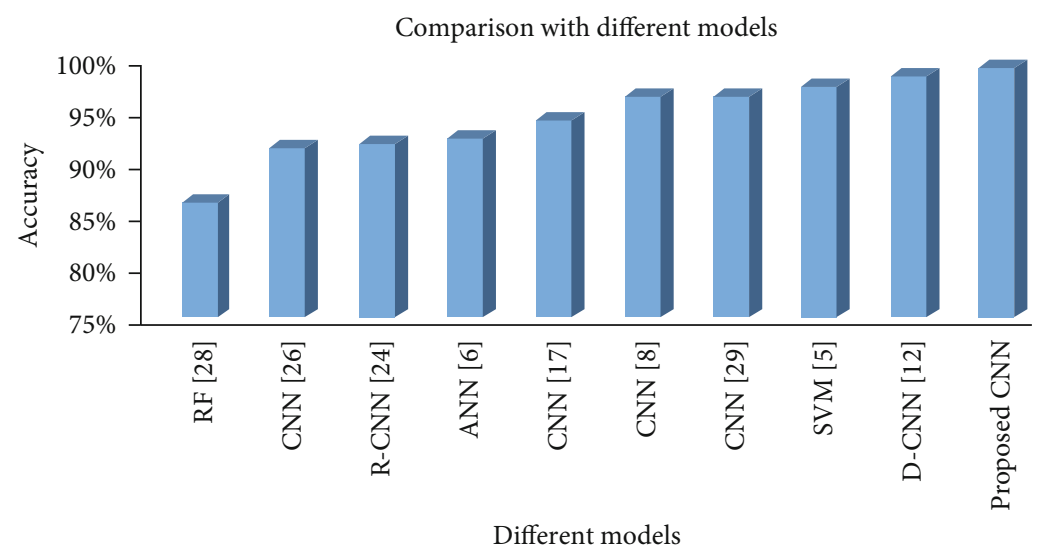

FIGURE 10: Comparison between the performance of the proposed CNN model and the other existing models.

based CNN (R-CNN) [35] are used. Table 4 gives an overview of the performance revealed by these systems. Least accuracy at $86 \%$ is shown by the Random Forest Classifier. Except for D-CNN, the accuracy achieved by CNN-based systems is between $91 \%$ and $96 \%$. Only D-CNN has achieved 98.07\% accuracy which is still below the accuracy that is revealed by the system proposed in this research. A clear comparison of the all these systems is portrayed in Table 5. Among these nine systems, six are CNN-based but still none of them is performing better than the system designed and proposed in this research. The CNN model with deep layers and data augmented MRIs has outperformed all the other systems as illustrated in Figure 10.

\section{Conclusion and Future Work}

In this research, a CNN based computer-aided diagnosis system of brain tumor is proposed. The deep network model $\mathrm{CNN}$ is trained only on $28 \%$ of data, and its performance is analysed on $72 \%$ of totally unseen data which is taken from different brain tumor MRI datasets. The model has provided, on average, $98.81 \%$ correct diagnosis of brain tumor while achieving $100 \%$ accuracy for two datasets. In the future, the performance of this CNN-based CAD system can be further enhanced by conducting further research and exploring other deep networks, variations of $\mathrm{CNN}$, feature maps, and augmentation techniques.

\section{Data Availability}

The datasets analysed during the current research are available at the links given below: (1) BR35H::Brain Tumor Detection 2020: https://www.kaggle.com/ahmedhamada0/ brain-tumor-detection. (2) Brain MRI Images for Brain Tumor Detection: https://www.kaggle.com/navoneel/brainmri-images-for-brain-tumor-detection. (3) Brain Tumor Images Dataset: https://www.kaggle.com/simeondee/braintumor-images-dataset. (4) Brain MRI Images for Brain Tumor Detection: https://www.kaggle.com/jjprotube/brainmri-images-for-brain-tumor-detection. (5) Brain Tumor Segmentation: https://www.kaggle.com/leaderandpiller/braintumor-segmentation. (6) test-brain: https://www.kaggle.com/ monagaffer12345/test-brain. (7) BD-BrainTumor: https://www .kaggle.com/dorianea/bd-braintumor

\section{Conflicts of Interest}

The authors declare that there is no conflict of interest. 


\section{References}

[1] J. J. Graber, C. S. Cobbs, and J. J. Olson, "Congress of Neurological Surgeons systematic review and evidence-based guidelines on the use of stereotactic radiosurgery in the treatment of adults with metastatic brain tumors," Neurosurgery, vol. 84, no. 3, pp. E168-E170, 2019.

[2] M. P. Brain, "Brain tumours: incidence, survival, and aetiology," Journal of Neurology, Neurosurgery and Psychiatry, vol. 75, no. 2, pp. 7-12, 2004.

[3] A. Patel, J. Fisher, E. Nichols, F. Abd-Allah, J. Abdela, and A. Abdelalim, "Global, regional, and national burden of brain and other CNS cancer, 1990-2016: a systematic analysis for the global burden of disease study," The Lancet Neurology, vol. 18, no. 4, pp. 93-376, 2019.

[4] K. P. Gliomas, "Survival, origin and early detection," Surgical Neurology International, vol. 1, 2011.

[5] K. J. Johnson, J. Schwartzbaum, C. Kruchko et al., "Brain tumor epidemiology in the era of precision medicine: the 2017 brain tumor epidemiology consortium meeting report," Clinical Neuropathology, vol. 36, no. 11, pp. 255-263, 2017.

[6] Y. M. Y. Abdallah and T. Alqahtani, "Research in medical imaging using image processing techniques," in Medical Imaging-Principles and Applications, IntechOpen, 2019.

[7] S. L. Fernandes, U. J. Tanik, V. Rajinikanth, and K. A. Karthik, "A reliable framework for accurate brain image examination and treatment planning based on early diagnosis support for clinicians," Neural Computing and Applications, vol. 32, no. 20, pp. 15897-15908, 2020.

[8] T. Saba, A. S. Mohamed, M. El-Affendi, J. Amin, and M. Sharif, "Brain tumor detection using fusion of hand crafted and deep learning features," Cognitive Systems Research, vol. 59, pp. 221-230, 2020.

[9] M. Alfonse and A. B. M. Salem, "An automatic classification of brain tumors through MRI using support vector machine," Egyptian Computer Science Journal, vol. 40, no. 3, 2016.

[10] A. K. Samanta and A. A. Khan, "Computer aided diagnostic system for automatic detection of brain tumor through MRI using clustering based segmentation technique and SVM classifier," in International Conference on Advanced Machine Learning Technologies and Applications, pp. 343-351, Cham, 2018.

[11] Z. N. K. Swati, Q. Zhao, M. Kabir et al., "Brain tumor classification for MR images using transfer learning and fine-tuning," Computerized Medical Imaging and Graphics, vol. 75, pp. 3446, 2019.

[12] K. Muhammad, S. Khan, J. Del Ser, and V. H. C. de Albuquerque, "Deep learning for multigrade brain tumor classification in smart healthcare systems: a prospective survey," IEEE Transactions on Neural Networks and Learning Systems, vol. 32, pp. 507-522, 2020.

[13] S. Deepak and P. Ameer, "Brain tumor classification using deep CNN features via transfer learning," Computers in Biology and Medicine, vol. 111, p. 103345, 2019.

[14] T. Hossain, F. S. Shishir, M. Ashraf, M. A. Al Nasim, and F. M. Shah, "Brain tumor detection using convolutional neural network," in 2019 1st International Conference on Advances in Science, Engineering and Robotics Technology (ICASERT), pp. 1-6, Dhaka, Bangladesh, 2019.

[15] M. Sajjad, S. Khan, K. Muhammad, W. Wu, A. Ullah, and S. W. Baik, "Multi-grade brain tumor classification using deep
CNN with extensive data augmentation," Journal of computational science, vol. 30, pp. 174-182, 2019.

[16] M. Toğaçar, B. Ergen, and Z. Cömert, "Brainmrnet: Brain tumor detection using magnetic resonance images with a novel convolutional neural network model," Medical Hypotheses, vol. 134, article 109531, 2020.

[17] A. Naseer and K. Zafar, "Comparative analysis of raw images and meta feature based Urdu OCR using CNN and LSTM," International Journal of Advanced Computer Science and Applications, vol. 9, no. 1, pp. 419-424, 2018.

[18] A. Naseer and K. Zafar, "Meta features-based scale invariant OCR decision making using LSTM-RNN," Computational and Mathematical Organization Theory, vol. 25, no. 2, pp. 165-183, 2019.

[19] A. Hamada, Br35h: Brain Tumor Detection 2020, version 5, 2020, https://www.kaggle.com/ahmedhamada0/brain-tumordetection.

[20] M. Ahmed, test_brain, version 1, 2020, https://www.kaggle .com/monagaffer12345/test-brain.

[21] N. Chakrabarty, Brain MRI images for brain tumor detection, version 1, 2019, https://www.kaggle.com/navoneel/brain-mriimages-for-brain-tumor-detection.

[22] J. Djhonson, Brain MRI images for brain tumor detection, version 1, 2020, https://www.kaggle.com/jjprotube/brainmri-images-for-brain-tumor-detection.

[23] A. Doriane, BD_Brain-Tumor, version 1, 2020, https://www .kaggle.com/dorianea/bd-braintumor.

[24] Leader, piller, Brain tumor segmentation, version 1, 2020, https://www.kaggle.com/leaderandpiller/braintumorsegmentation.

[25] A. Simeon, Brain tumor images dataset, version 1, 2019, https:// www.kaggle.com/simeondee/braintumor-images-dataset.

[26] F. Özyurt, E. Sert, E. Avci, and E. Dogantekin, "Brain tumor detection based on convolutional neural network with neutrosophic expert maximum fuzzy sure entropy," Measurement, vol. 147, p. 106830, 2019.

[27] J. Amin, M. Sharif, N. Gul, M. Yasmin, and S. A. Shad, "Brain tumor classification based on DWT fusion of MRI sequences using convolutional neural network," Pattern Recognition Letters, vol. 129, pp. 115-122, 2020.

[28] E. Dandil and S. Karaca, "Detection of pseudo brain tumors via stacked LSTM neural networks using MR spectroscopy signals," Biocybernetics and Biomedical Engineering, vol. 41, no. 1, pp. 173-195, 2021.

[29] F. J. Díaz-Pernas, M. Martínez-Zarzuela, M. Antón-Rodríguez, and D. González-Ortega, "A deep learning approach for brain tumor classification and segmentation using a multiscale convolutional neural network," in Healthcare, vol. 9, p. 153, Multidisciplinary Digital Publishing Institute, 2021.

[30] R. L. Kumar, J. Kakarla, B. V. Isunuri, and M. Singh, "Multiclass brain tumor classification using residual network and global average pooling," Multimedia Tools and Applications, vol. 80, no. 9, pp. 13429-13438, 2021.

[31] T. Kalaiselvi and S. Padmapriya, "Brain tumor diagnostic system-a deep learning application," in Machine Vision Inspection Systems, vol. 2, pp. 69-90, Machine LearningBased Approaches, 2021.

[32] A. Rehman, M. A. Khan, T. Saba, Z. Mehmood, U. Tariq, and N. Ayesha, "Microscopic brain tumor detection and classification using 3D CNN and feature selection architecture," Microscopy Research and Technique, vol. 84, no. 1, pp. 133-149, 2021. 
[33] C. L. Choudhury, C. Mahanty, R. Kumar, and B. K. Mishra, "Brain tumor detection and classification using convolutional neural network and deep neural network," in 2020 International Conference on Computer Science, Engineering and Applications (ICCSEA), pp. 1-4, Gunupur, India, 2020.

[34] M. L. Martini and E. K. Oermann, "Intraoperative brain tumour identification with deep learning," Nature Reviews Clinical Oncology, vol. 17, no. 4, pp. 200-201, 2020.

[35] E. Avşar and K. Salçin, "Detection and classification of brain tumours from MRI images using faster R-CNN," Tehnički glasnik, vol. 13, no. 4, pp. 337-342, 2019.

[36] S. Sarkar, A. Kumar, S. Chakraborty, S. Aich, J. S. Sim, and H. C. Kim, "A CNN based approach for the detection of brain tumor using MRI scans," Test Engineering and Management, pp. 16580-16586, 2020.

[37] H. H. Sultan, N. M. Salem, and W. Al-Atabany, "Multi-classification of brain tumor images using deep neural network," IEEE Access, vol. 7, pp. 69215-69225, 2019.

[38] M. Soltaninejad, G. Yang, T. Lambrou et al., "Supervised learning based multimodal MRI brain tumour segmentation using texture features from supervoxels," Computer Methods and Programs in Biomedicine, vol. 157, pp. 69-84, 2018.

[39] N. Arunkumar, M. A. Mohammed, S. A. Mostafa, D. A. Ibrahim, J. J. Rodrigues, and V. H. C. de Albuquerque, "Fully automatic model-based segmentation and classification approach for MRI brain tumor using artificial neural networks," Concurrency and Computation: Practice and Experience, vol. 32, no. 1, article e4962, 2020.

[40] M. Ganesan, N. Sivakumar, and M. Thirumaran, "Internet of medical things with cloud-based E-health services for brain tumour detection model using deep convolution neural network," Electronic Government, an International Journal, vol. 16, no. 1/2, pp. 69-83, 2020.

[41] J. Amin, M. Sharif, M. Yasmin, and S. L. Fernandes, “A distinctive approach in brain tumor detection and classification using MRI," Pattern Recognition Letters, vol. 139, 2017. 